\title{
ELSEVIER_BIOC_5770
}

\section{Modelling the benefits of habitat restoration in socio-ecological systems}

Sacha Jellinek ${ }^{a *}$

sacha.jellinek@gmail.com

Libby Rumpff ${ }^{a}$

Don A. Driscoll

Kirsten M. Parris

Brendan A. Wintle ${ }^{a}$

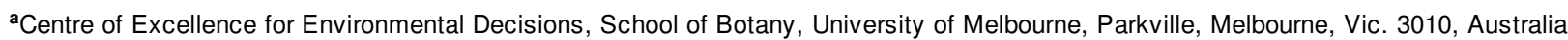

' $T$ The Fenner School of Environment and Society, Australian National University, Hancock Building (43), Biology Place, Canberra, ACT 0200

Australia

\section{Abstract}

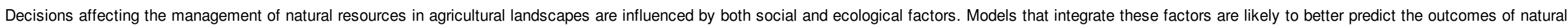

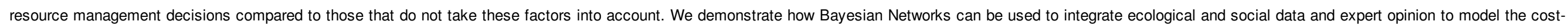

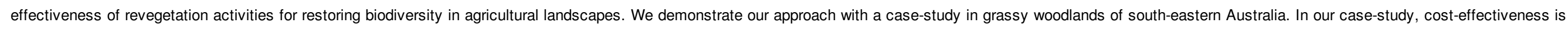

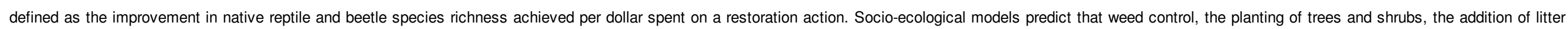

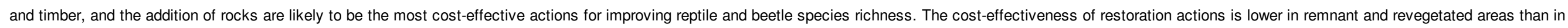

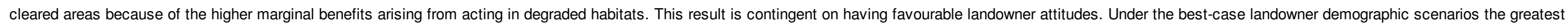

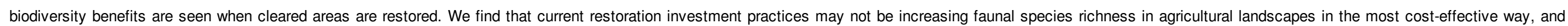

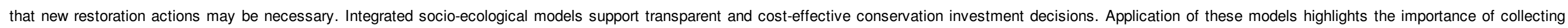
both social and ecological data when attempting to understand and manage socio-ecological systems.

Keywords: Bayesian Networks; Socio-ecological systems; Expert opinion; Restoration; Revegetation; Decision making; Uncertainty; Species richness

\section{Introduction}

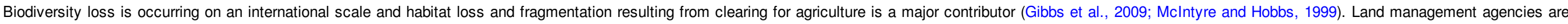

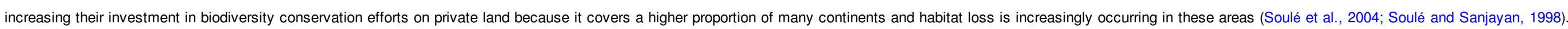

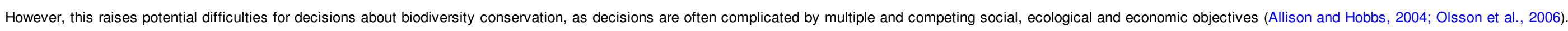

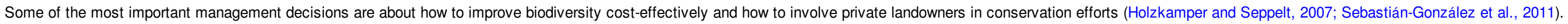

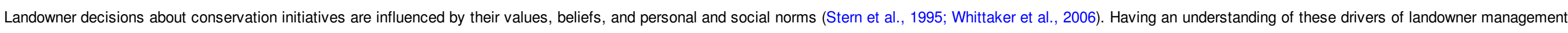

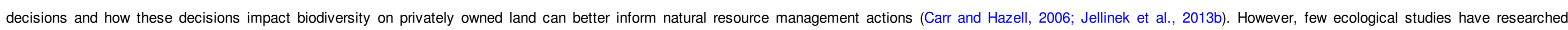

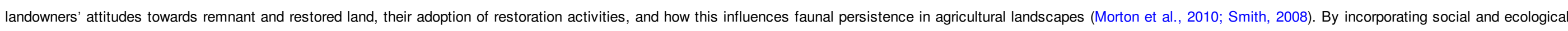

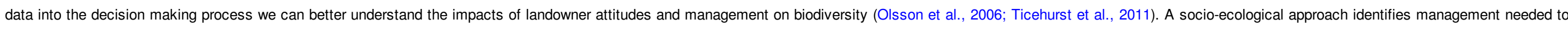




\section{ELSEVIER_BIOC_5770}

achieve conservation objectives, and defines the social constraints and opportunities for implementing that management (Wyborn et al., 2012).

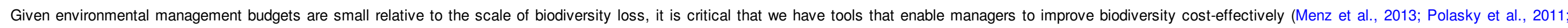

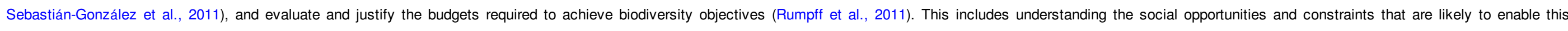

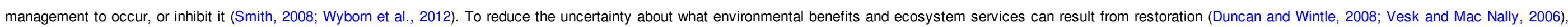
there is also a need to explicitly calculate the cost-effectiveness of competing restoration options (Measham, 2007; Rumpff et al., 2011).

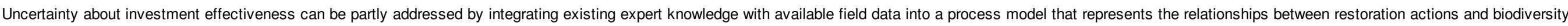

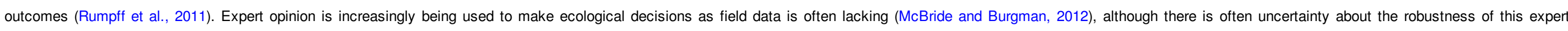

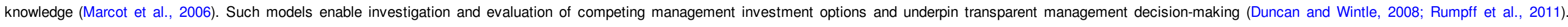

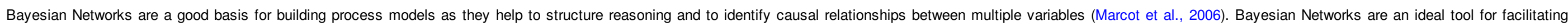

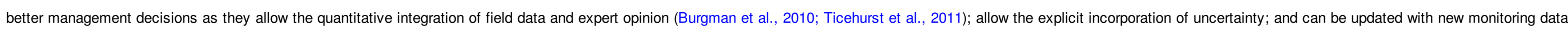
to reflect a better understanding of the natural system over time (Glendining and Pollino, 2012; Rumpff et al., 2011).

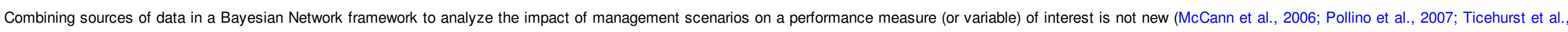

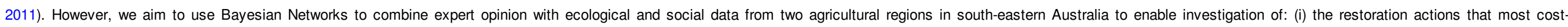

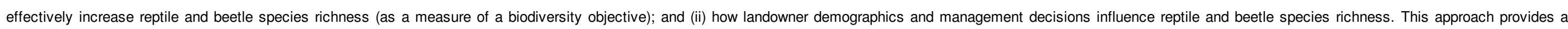
useful tool for management agencies attempting to understand the social opportunities and constraints associated with making cost-effective decisions about biodiversity conservation in agricultural landscapes.

\section{Materials and methods}

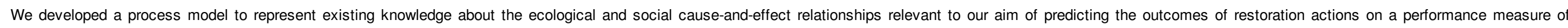

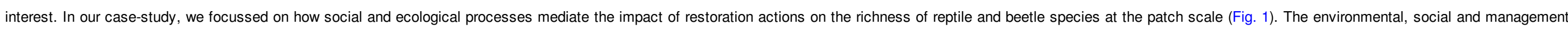

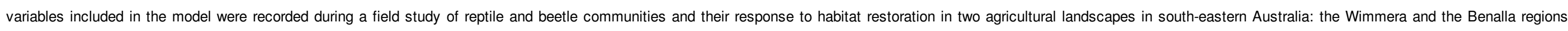
(Jellinek et al., 2013a, 2013b, in preparation).

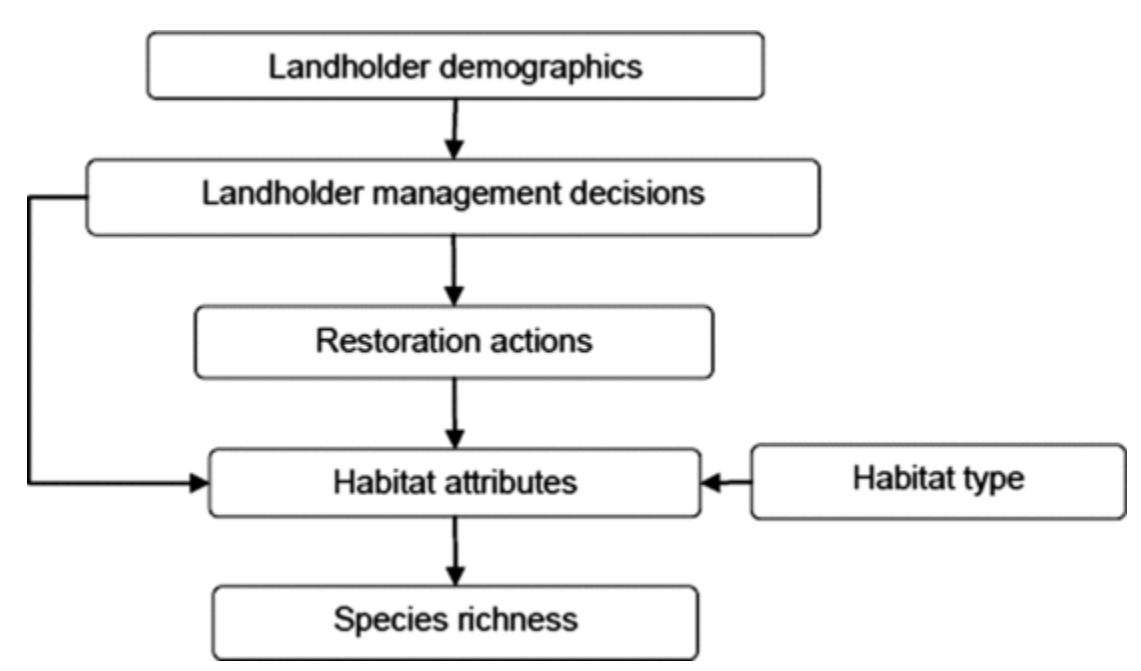

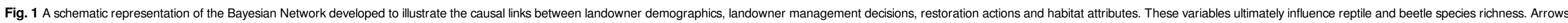
represent a flow of information from one group of variables to another. 


\section{ELSEVIER_BIOC_5770}

\subsection{Data collection}

\subsubsection{Study area}

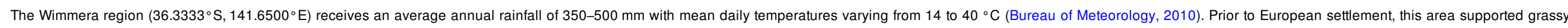

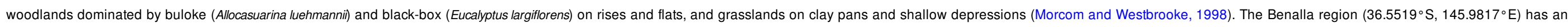

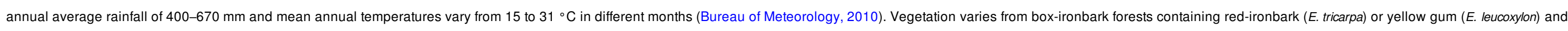

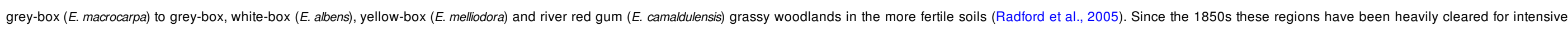

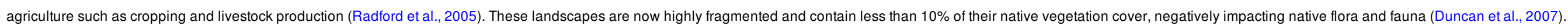

\subsubsection{Landowner data}

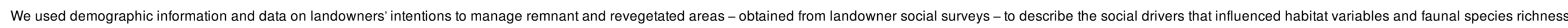

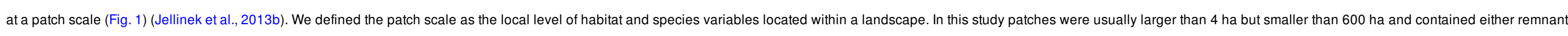
or restored habitat. This differs to landscape scale processes that incorporate multiple patch scale processes (Graham and Blake, 2001).

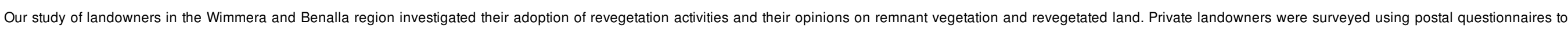

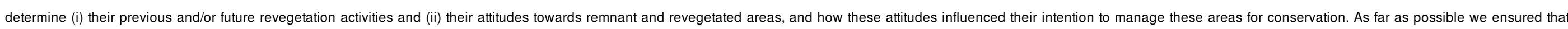

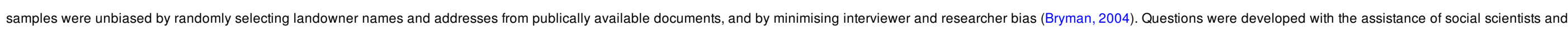

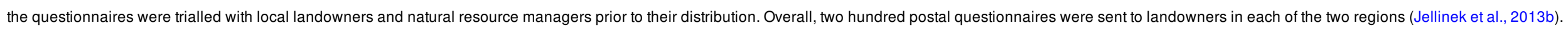

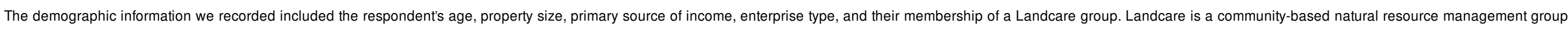

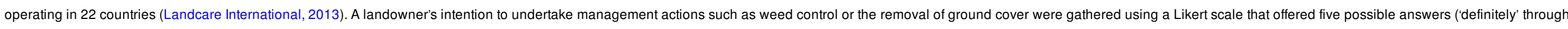

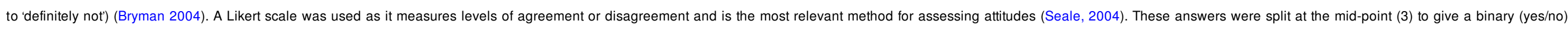

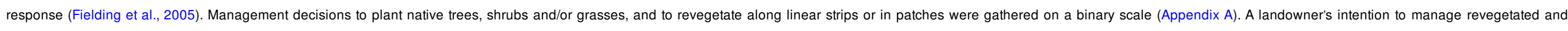
remnant areas was a function of their attitudes to these areas.

\subsubsection{Ecological data}

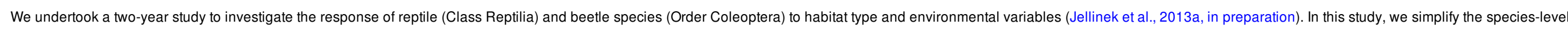

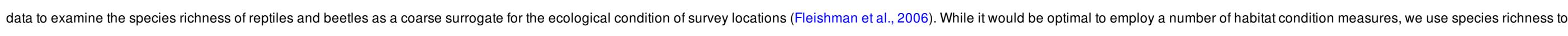

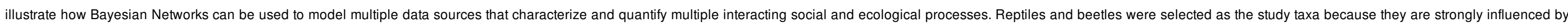
vegetation type and structure (Mac Nally and Brown, 2001; Schaffers et al., 2008) but are seldom studied in the restoration literature (Munro et al., 2007).

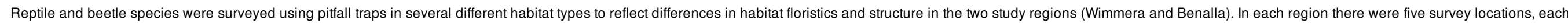

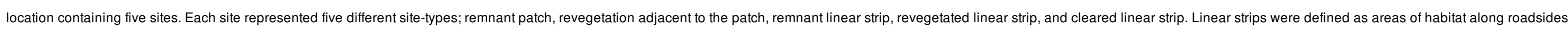

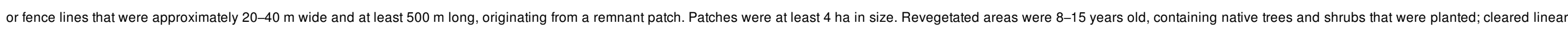

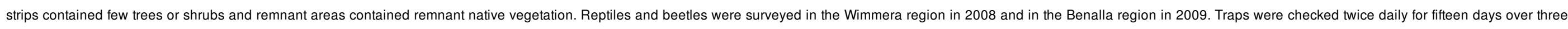
months (January-March) in each of the two years.

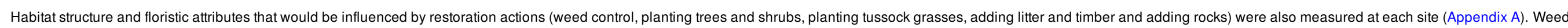

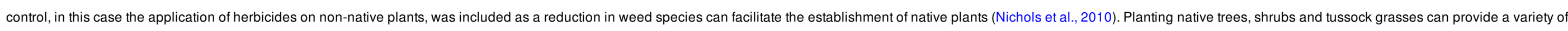

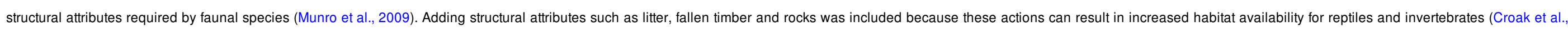
2010; Grimbacher and Catterall, 2007). 


\section{ELSEVIER_BIOC_5770}

\subsection{Modelling framework}

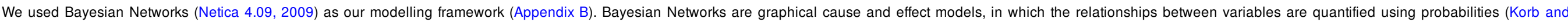

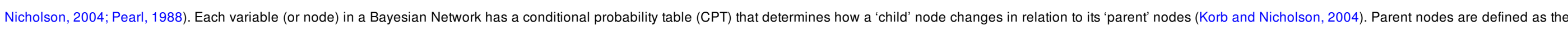

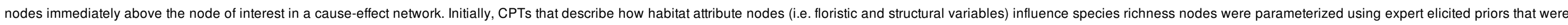

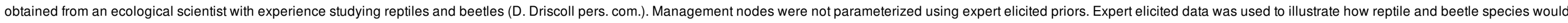
be expected to respond to changes in habitat floristics and structure when field data were absent.

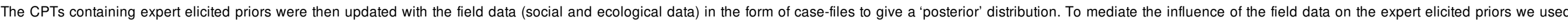

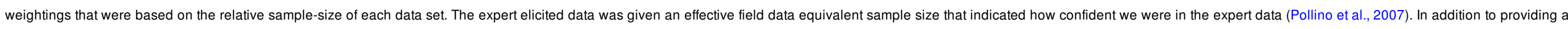

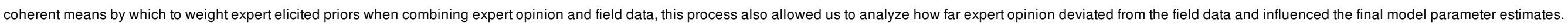

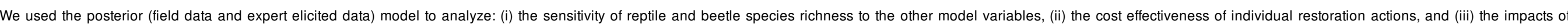
landowner demographic scenarios on biodiversity outcomes.

\subsection{Data analysis}

\subsubsection{Sensitivity to findings}

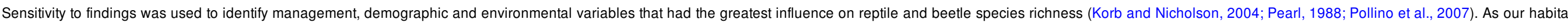

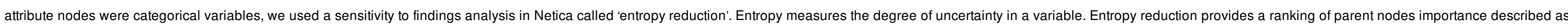

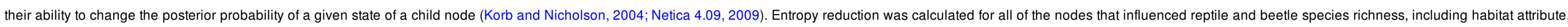
landowner demographic and landowner management decision nodes.

\subsubsection{Cost-effectiveness and demographic scenarios}

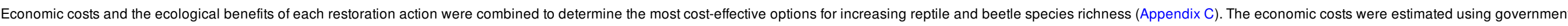

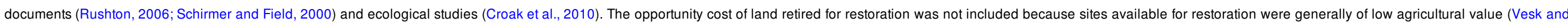
Mac Nally, 2006), and because restoration may have provided future economic benefits to the landowner (Dorrough et al., 2008).

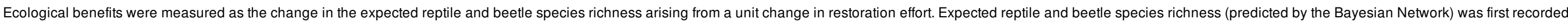

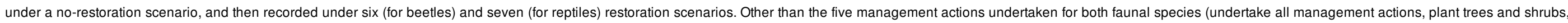

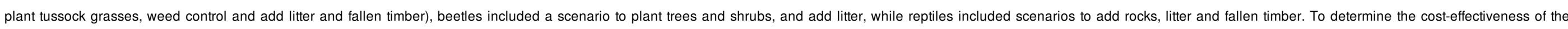

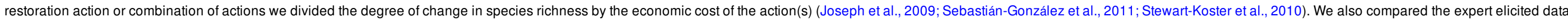
with the updated expert and field data model to calculate how expert opinion compared to field data.

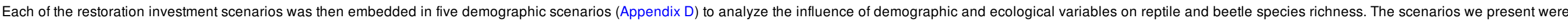

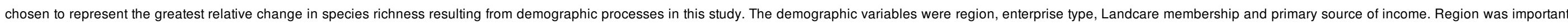

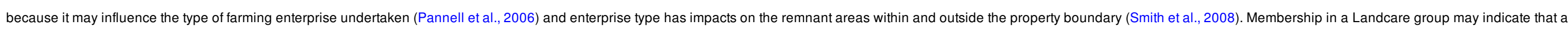
landowner is more likely to carry out conservation actions (Curtis and De Lacy, 1996), while an off-farm income can increase a landowner's ability and propensity to undertake conservation activities (Pannell et al., 2006).

\section{Results}

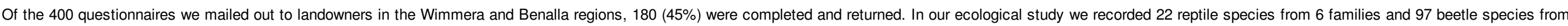




\section{ELSEVIER_BIOC_5770}

10 families.

\subsection{Sensitivity to findings}

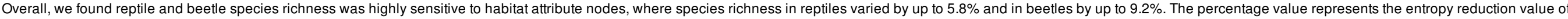

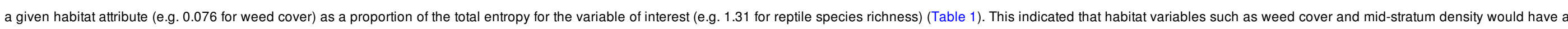

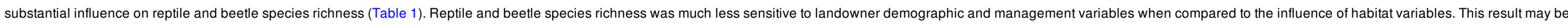

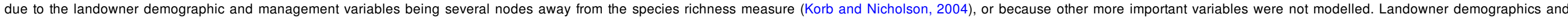
management variables to remove litter and timber and replant trees and shrubs had a stronger positive influence on species richness than other management decisions (Table 1).

Table 1 Sensitivity analysis using combined expert and field data models showing reptile and beetle species richness sensitivity to habitat attributes, landowner demographics and landowner management decisions.

\begin{tabular}{|c|c|c|}
\hline Variables & Reptile species richness & Beetle species richness \\
\hline Habitat attributes & Entropy $(Q)=1.31$ & Entropy $(Q)=15.65$ \\
\hline Weed cover & $0.076(5.8 \%)$ & $1.434(9.2 \%)$ \\
\hline Mid-stratum density & $0.053(4 \%)$ & $1.409(9 \%)$ \\
\hline Litter cover & $0.048(3.6 \%)$ & $0.692(4.5 \%)$ \\
\hline Tussock cover & $0.016(1.2 \%)$ & $0.158(1 \%)$ \\
\hline Herb cover & n/a & $0.115(0.7 \%)$ \\
\hline Rock cover & $0.018(1.3 \%)$ & n/a \\
\hline Landowner demographics and management decisions & Entropy $(Q)=1.31$ & Entropy $(Q)=15.46$ \\
\hline Region & $0.0002(0.02 \%)$ & $0.0019(0.01 \%)$ \\
\hline Landcare membership & $0.0000(0.01 \%)$ & $0.0016(0.01 \%)$ \\
\hline Income & $0.0001(0.01 \%)$ & $0.0006(0.00 \%)$ \\
\hline Enterprise type & $0.0003(0.03 \%)$ & $0.0014(0.01 \%)$ \\
\hline Remove litter and timber & $0.0053(0.41 \%)$ & $0.0375(0.24 \%)$ \\
\hline Revegetate trees and shrubs & $0.0033(0.17 \%)$ & $0.0329(0.21 \%)$ \\
\hline Revegetate tussocks & $0.0007(0.05 \%)$ & $0.0002(0.00 \%)$ \\
\hline Weed management & $0.0006(0.05 \%)$ & $0.0053(0.04 \%)$ \\
\hline Revegetation type & $0.0003(0.02 \%)$ & $0.0021(0.01 \%)$ \\
\hline
\end{tabular}

\subsection{Cost-effectiveness}

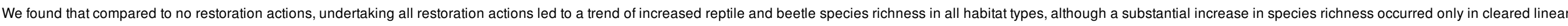

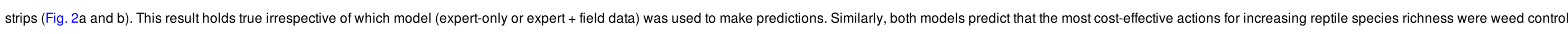

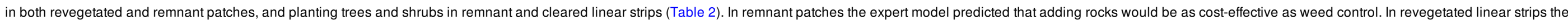

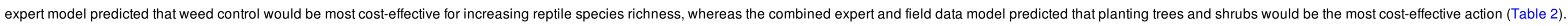

\section{${ }_{0}{ }^{4}$ (a)


ELSEVIER_BIOC_5770
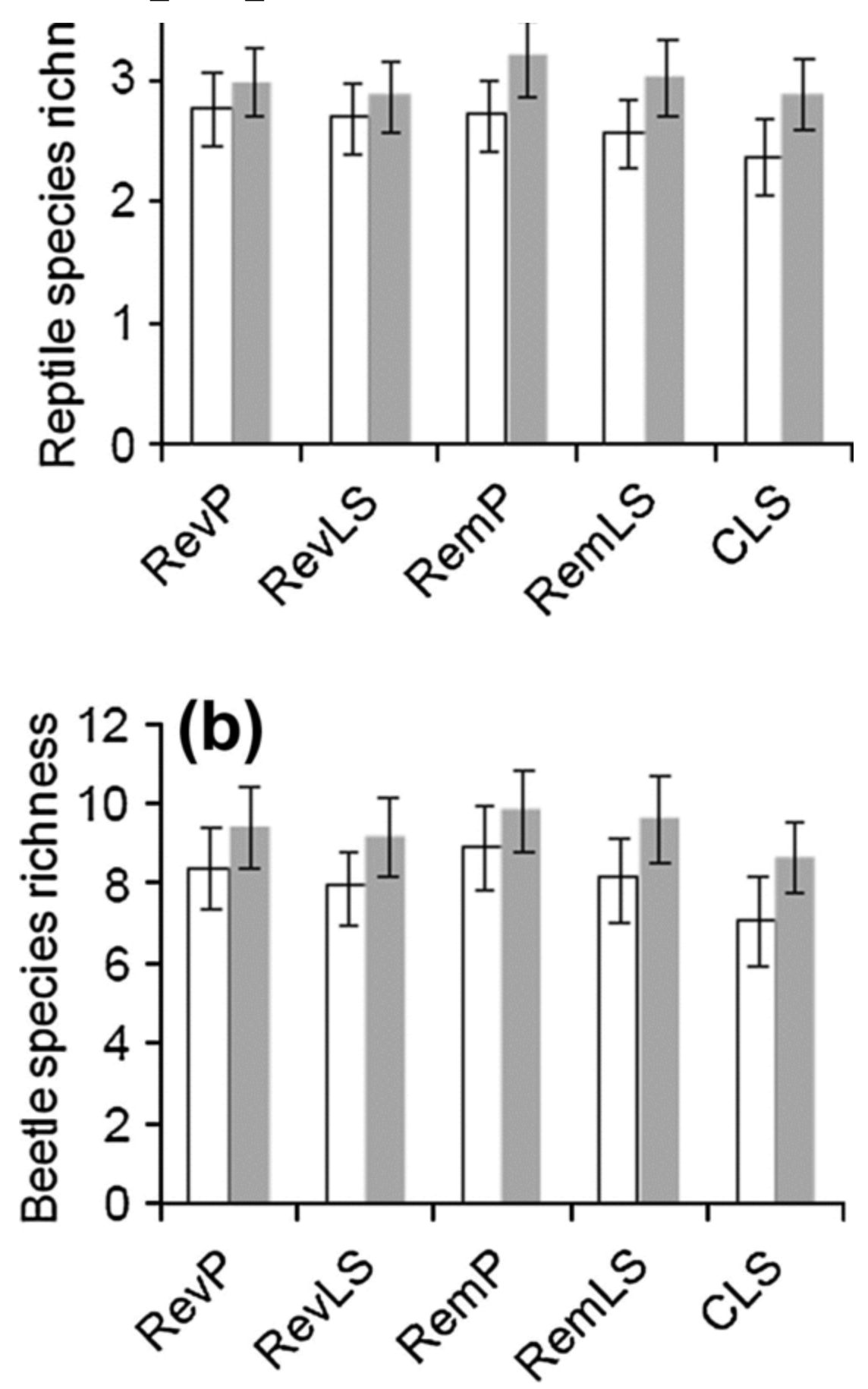

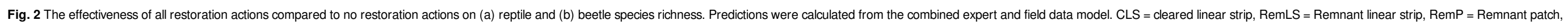




\section{ELSEVIER_BIOC_5770}

RevLS $=$ Revegetated linear strip. Clear columns $=$ no restoration, grey columns $=$ all restoration actions. Bars represent $95 \%$ credible intervals, which are analogous to frequentist confidence intervals.

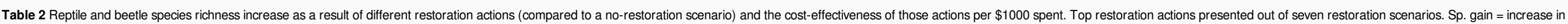

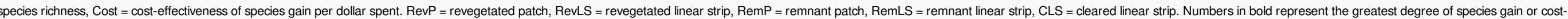
effectiveness for each restoration action.

\begin{tabular}{|c|c|c|c|c|c|c|c|c|c|}
\hline \multirow[t]{3}{*}{ Habitat type } & \multirow[t]{3}{*}{ Restoration actions } & \multicolumn{4}{|c|}{ Reptile species richness } & \multicolumn{4}{|c|}{ Beetle species richness } \\
\hline & & \multicolumn{2}{|c|}{ Expert model } & \multicolumn{2}{|c|}{ Combined expert and field model } & \multicolumn{2}{|c|}{ Expert model } & \multicolumn{2}{|c|}{ Combined expert and field model } \\
\hline & & Sp. gain & Cost & Sp. gain & Cost & Sp. gain & Cost & Sp. gain & Cost \\
\hline RevP & All restoration & 0.47 & 0.06 & 0.22 & 0.03 & 0.57 & 0.07 & 1.06 & 0.13 \\
\hline RevP & Weed control & 0.08 & 0.42 & 0.06 & 0.32 & 0.24 & 1.26 & 0.03 & 0.16 \\
\hline RevP & Add rocks and litter & 0.41 & 0.09 & 0.39 & 0.09 & - & - & - & - \\
\hline RevP & Add litter & - & - & - & - & 0.47 & 0.16 & 0.51 & 0.17 \\
\hline RevLS & All restoration & 0.42 & 0.05 & 0.52 & 0.06 & 0.61 & 0.07 & 1.30 & 0.15 \\
\hline RevLS & Weed control & 0.08 & 0.42 & -0.12 & -0.63 & 0.25 & 1.32 & -0.06 & -0.32 \\
\hline RevLS & Plant trees and shrubs & -0.06 & -0.07 & 0.29 & 0.36 & - & - & - & - \\
\hline RevLS & Add litter & - & - & - & - & 0.47 & 0.16 & 0.68 & 0.23 \\
\hline RemP & All restoration & 0.61 & 0.07 & 0.47 & 0.06 & 1.34 & 0.16 & 0.92 & 0.11 \\
\hline RemP & Weed control & 0.03 & 0.16 & 0.02 & 0.11 & 0.10 & 0.53 & 0.10 & 0.53 \\
\hline RemP & Add rocks & 0.24 & 0.16 & 0.16 & 0.05 & - & - & - & - \\
\hline RemLS & All restoration & 0.61 & 0.07 & 0.45 & 0.05 & 2.15 & 0.25 & 1.51 & 0.18 \\
\hline RemLS & Plant trees and shrubs & 0.16 & 0.20 & 0.13 & 0.16 & 1.30 & 1.59 & 0.80 & 0.98 \\
\hline CLS & All restoration & 0.86 & 0.10 & 0.52 & 0.06 & 3.93 & 0.46 & 1.61 & 0.19 \\
\hline CLS & Plant trees and shrubs & 0.30 & 0.37 & 0.29 & 0.36 & 2.87 & 3.52 & 0.28 & 0.34 \\
\hline CLS & Add Litter & - & - & - & - & 0.20 & 0.07 & 1.30 & 0.44 \\
\hline
\end{tabular}

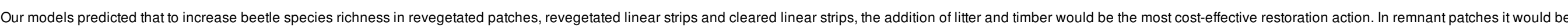
weed control, and in remnant linear strips planting trees and shrubs would be most cost-effective (Table 2).

\subsection{Landowner demographic scenarios}

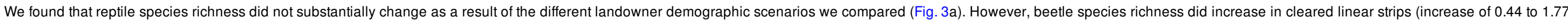

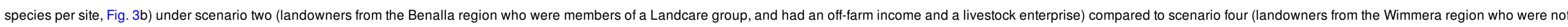

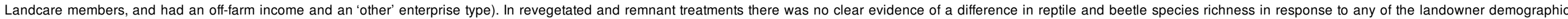
scenarios (Fig. 3a and b).

(a)

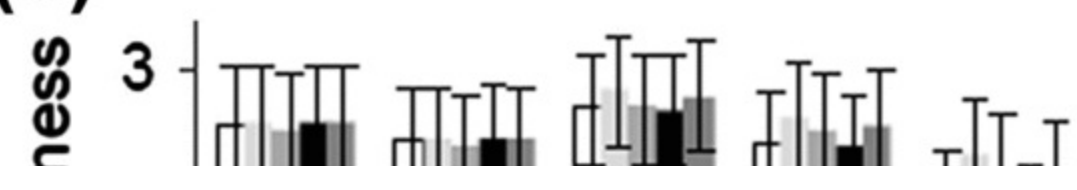




\section{ELSEVIER_BIOC_5770}

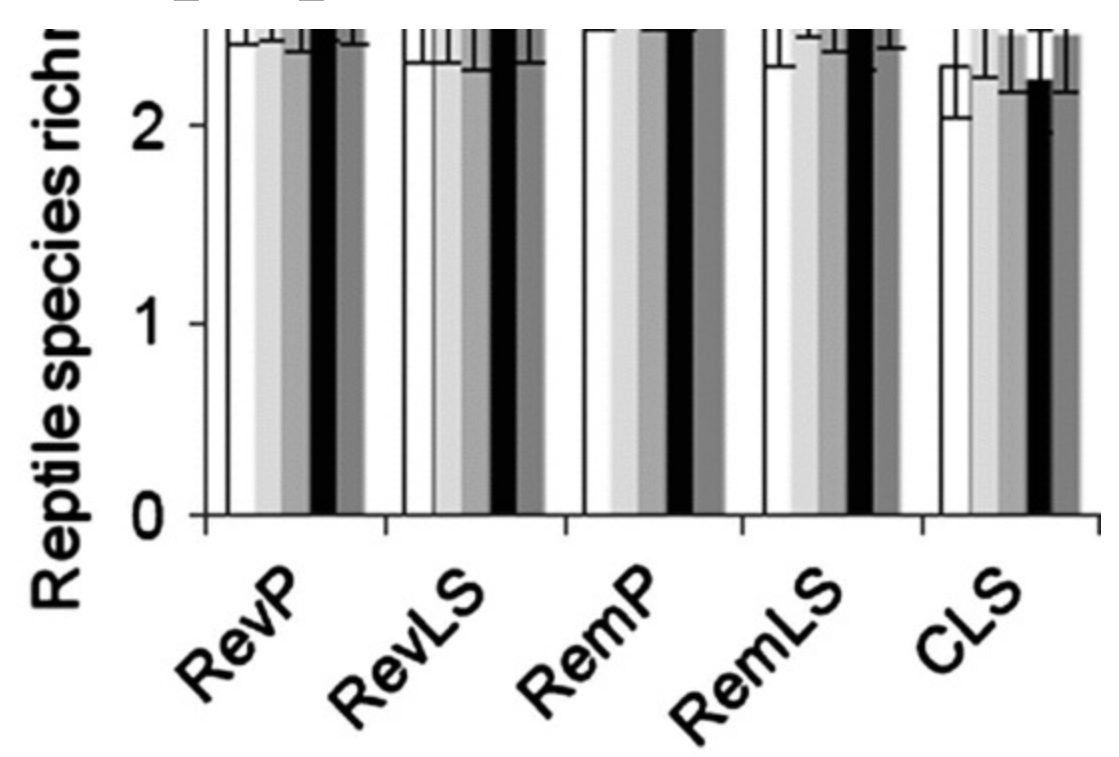

(b)

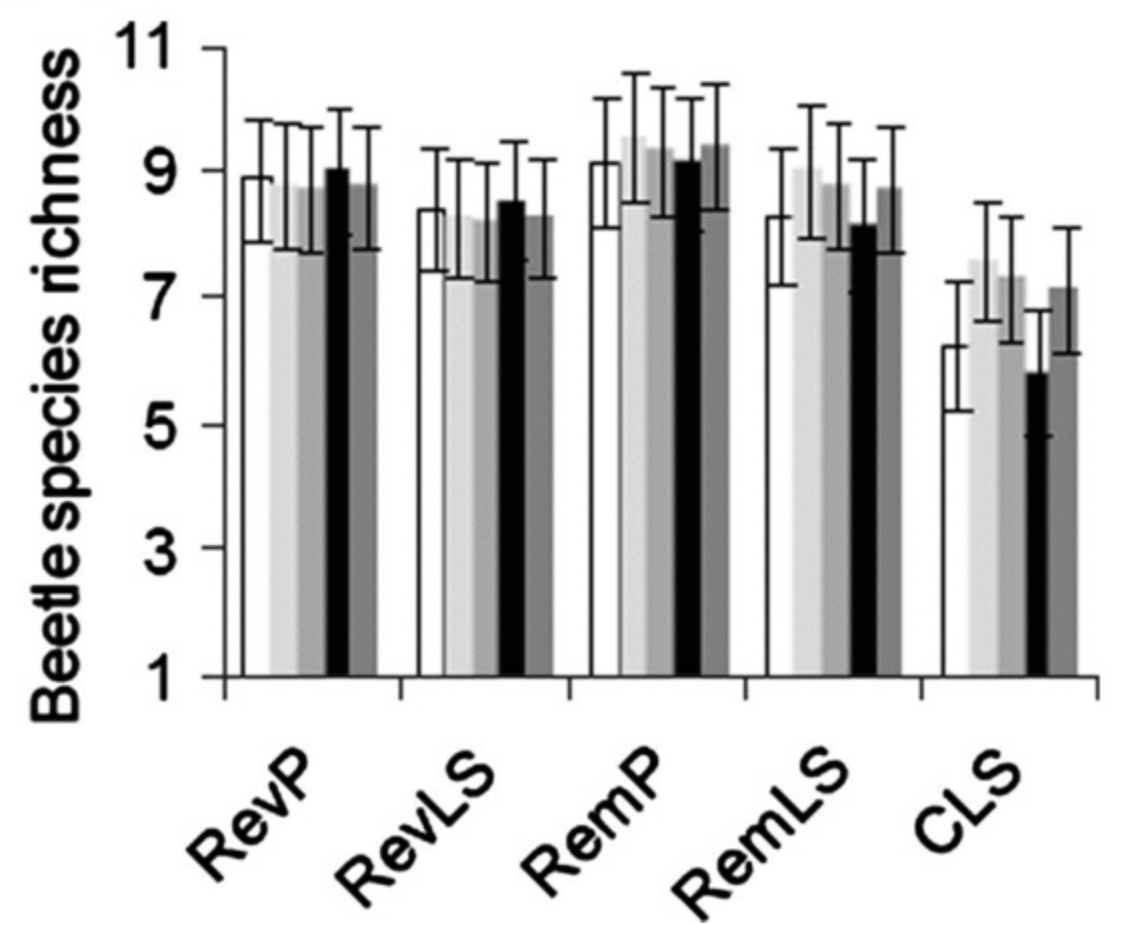

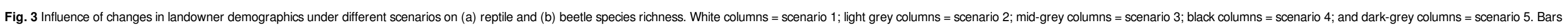




\section{Discussion}

\subsection{Integrating social and ecological data in a cost-effectiveness analysis}

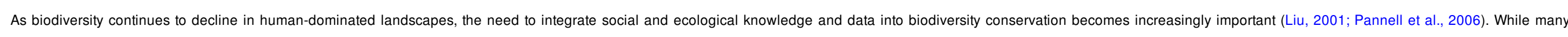

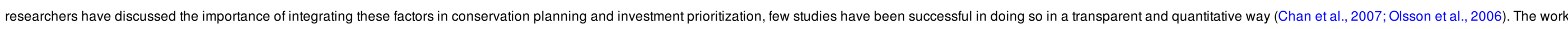

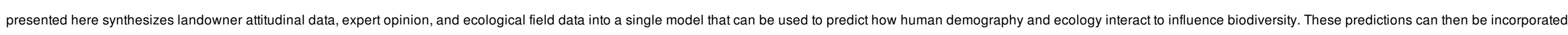
in feasibility and cost-effectiveness analyses to help support conservation investment decisions that explicitly address uncertainty

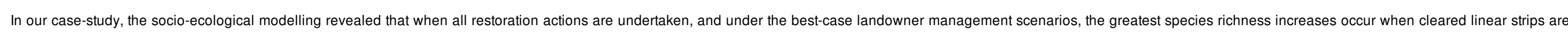

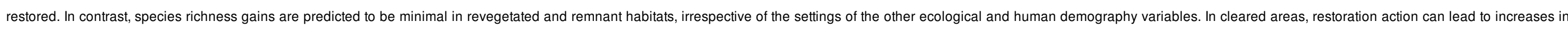

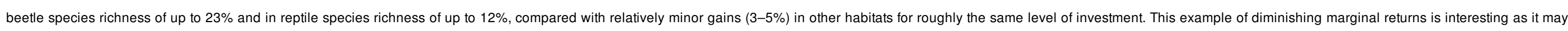

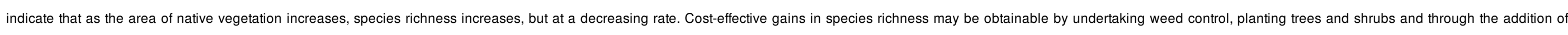

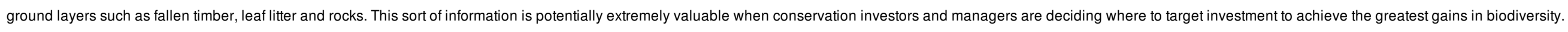

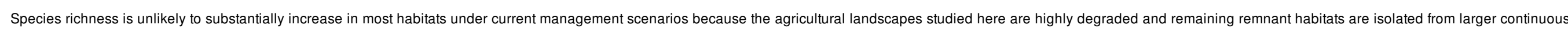

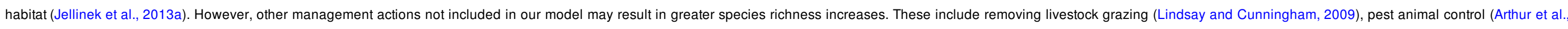
2010), creating connections and broad-scale revegetation (Munro et al., 2007). The cost-effectiveness of these actions should be quantified as a priority to measure the relative biodiversity benefits of these actions.

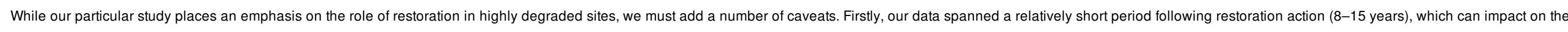

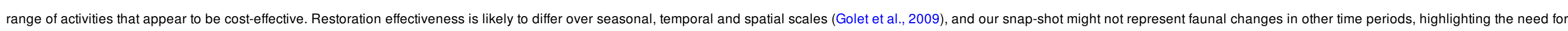

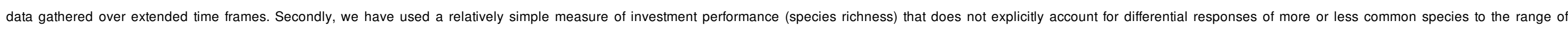
conservation actions considered (Fleishman et al., 2006). Nonetheless, we argue that the analytical approach we describe here can inform management decisions and cost-effectively maintain biodiversity.

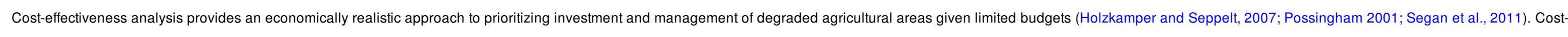

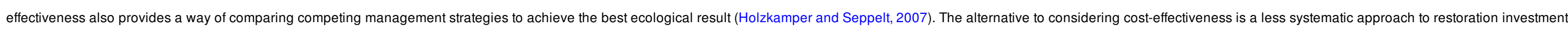
prioritization that may be ineffective in maintaining biodiversity and inappropriately allocate conservation funding (Lunney et al., 1997).

\subsection{Expert opinion and field data}

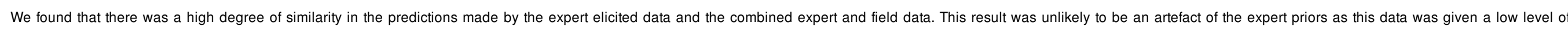

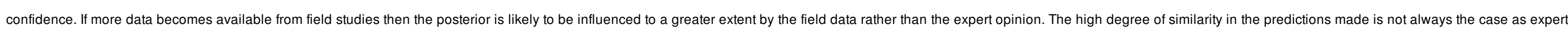

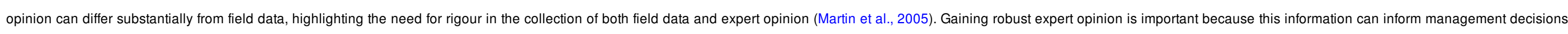
when ecological data are lacking (Kuhnert et al., 2010; Marcot et al., 2006; McBride and Burgman, 2012).

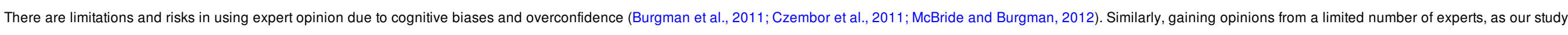

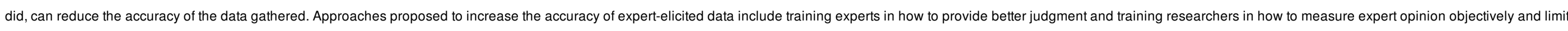

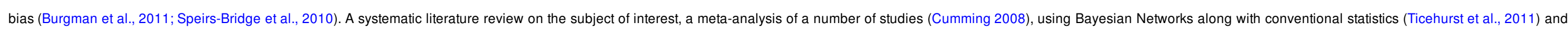
using multiple experts may also increase information accuracy.

\subsection{Future research}

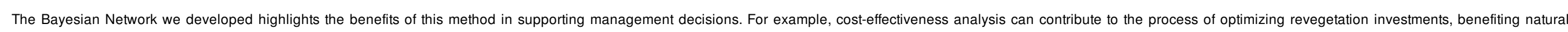




\section{ELSEVIER_BIOC_5770}

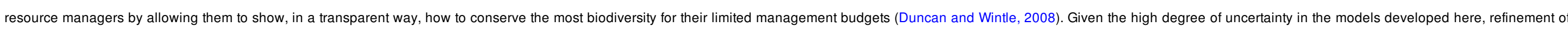

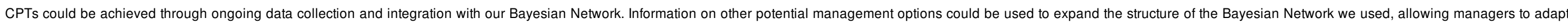

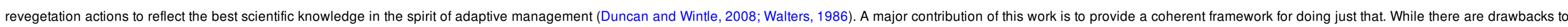
this method, such as subjectivity in structuring and parameterizing the Bayesian Network, these can be addressed systematically using rigorous expert elicitation processes.

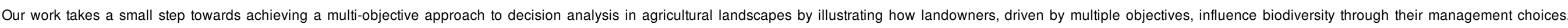

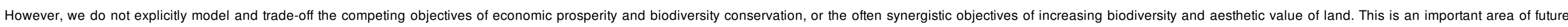
research that can be partly informed by what has been shown here.

\section{Conclusion}

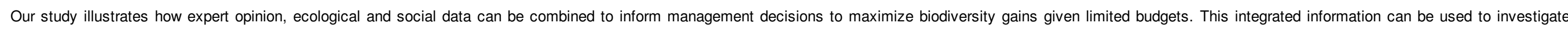

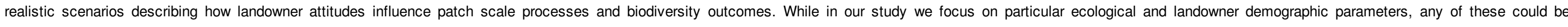

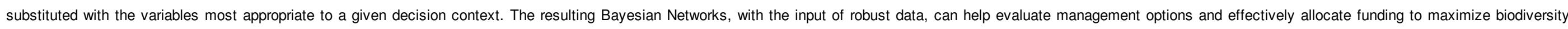

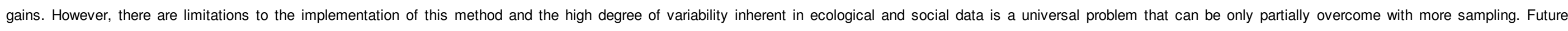

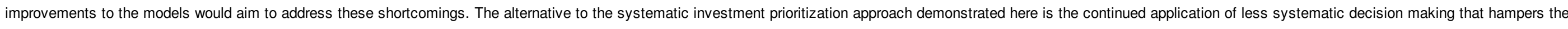
ongoing refinement of restoration knowledge and progress toward effective conservation decision making.

\section{Acknowledgements}

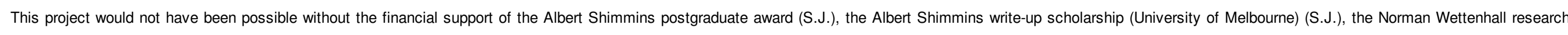

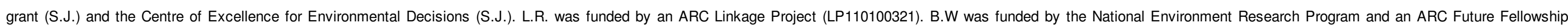
(FT100100819). D.D. was supported by the National Environmental Research Program and the Centre of Excellence for Environmental Decisions.

\section{Appendix A. Supplementary material}

Supplementary data associated with this article can be found, in the online version, at http://dx.doi.org/10.1016/j.biocon.2013.10.023.

\section{References}

Allison H.E. and Hobbs R.J., Resilience, adaptive capacity, and the "Lock-in Trap" of the Western Australian agricultural region, Ecol. Soc. 9, $2004,3$.

Arthur A.D., Henry S. and Reid A., Influence of revegetation on predation rates by introduced red foxes (Vulpes vulpes) in south-eastern Australian farmland, Austral Ecol. 35, $2010,919-928$.

Bryman A., Social Research Methods, 2nd ed., 2004, Oxford University Press; New York.

Bureau of Meteorology, 2010. Bureau of Meteorology, Commonwealth of Australia.

Burgman M., Carr A., Godden L., Gregory R., McBride M., Flander L. and Maguire L., Redefining expertise and improving ecological judgment, Conserv. Lett. 4, 2011, 81-87.

Burgman M.A., Wintle B.A., Thompson C.A., Moilanen A., Runge M.C. and Ben-Haim Y., Reconciling uncertain costs and benefits in Bayes Nets for invasive species management, Risk Anal. 30, $2010,277-284$.

Carr A. and Hazell D., Talking frogs: the role of communication in ecological research on private land, Biodivers. Conserv. 15, $2006,3177-3191$.

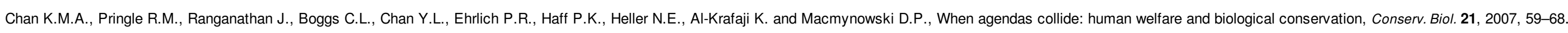
Croak B.M., Pike D.A., Webb J.K. and Shine R., Using artificial rocks to restore nonrenewable shelter sites in human-degraded systems: colonization by fauna, Restor. Ecol. 18, 2010 , 428-438.

Cumming G., Replication and $p$ intervals $p$ values predict the future only vaguely, but confidence intervals do much better, Perspect. Psychol. Sci. 3, 2008, 286-300. 


\section{ELSEVIER_BIOC_5770}

Curtis A. and De Lacy T., Landcare in Australia: does it make a difference?, J. Environ. Manage. 46, 1996, 119-137.

Czembor C.A., Morris W.K., Wintle B.A. and Vesk P.A., Quantifying variance components in ecological models based on expert opinion, J. Appl. Ecol. 48, 2011, 736-745.

Dorrough J., Vesk P.A. and Moll J., Integrating ecological uncertainty and farm-scale economics when planning restoration, J. Appl. Ecol. 45, 2008, $288-295$.

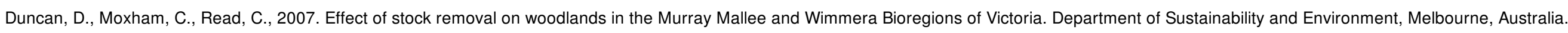

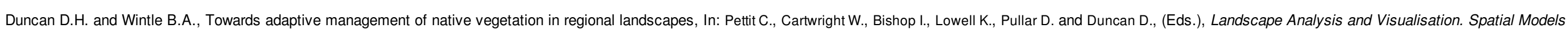

for Natural Resource Management and Planning, 2008, Springer-Verlag; Berlin, 159-182.

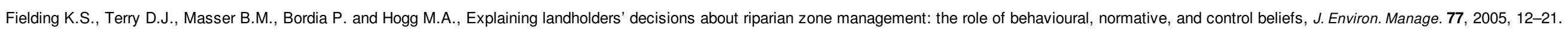

Fleishman E., Noss R.F. and Noon B.R., Utility and limitations of species richness metrics for conservation planning, Ecol. Indic. 6, 2006, 543-553.

Gibbs K.E., Mackey R.L. and Currie D.J., Human land use, agriculture, pesticides and losses of imperiled species, Divers. Distrib. 15, 2009, 242-253.

Glendining N.S. and Pollino C.A., Development of Bayesian network decision support tools to support river rehabilitation works in the Lower Snowy River, Human Ecol. Risk. Assess. Int. J. 18, 2012, 92-114.

Golet G.H., Gardali T., Hunt J.W., Koenig D.A. and Williams N.M., Temporal and taxonomic variability in response of fauna to riparian restoration, Restor. Ecol. 2009 , 1-10.

Graham C.H. and Blake J.G., Influence of patch- and landscape-level factors on bird assemblages in a fragmented tropical landscape, Ecol. Appl. 11, 2001, 1709-1721.

Grimbacher P.S. and Catterall C.P., How much do site age, habitat structure and spatial isolation influence the restoration of rainforest beetle species assemblages?, Biol. Conserv. 135, 2007, 107-118.

Holzkamper A. and Seppelt R., Evaluating cost-effectiveness of conservation management actions in an agricultural landscape on a regional scale, Biol. Conserv. 136, $2007,117-127$.

Jellinek S., Parris K.P. and Driscoll D.A., Are only the strong surviving? Little influence of restoration on beetles (Coleoptera) in an agricultural landscape, Biol. Conserv. 162, 2013a, 17-23.

Jellinek S., Parris K.P., Driscoll D.A. and Dwyer P.D., Are incentive programs working? Landowner attitudes to ecological restoration of agricultural landscapes, J. Environ. Manage. 127, $2013 b, 69-76$.

Jellinek, S., Parris, K.P., Wintle, B.A., Driscoll, D.A., in revision. Reptiles in restored agricultural landscapes: The value of linear strips, patches and habitat condition. Anim Conserv Joseph L.N.,

Maloney R.F. and Possingham H.P., Optimal allocation of resources among threatened species: a project prioritization protocol, Conserv. Biol. 23, 2009, 328-338.

Korb K.B. and Nicholson A.E., Bayesian Artificial Intelligence, 2004, Chapman and Hall/CRC Press; London, UK.

Kuhnert P.M., Martin T.G. and Griffiths S.P., A guide to eliciting and using expert knowledge in Bayesian ecological models, Ecol. Lett. 13, 2010, 900-914.

Landcare International, 2013.

Lindsay E.A. and Cunningham S.A., Livestock grazing exclusion and microhabitat variation affect invertebrates and litter decomposition rates in woodland remnants, For. Ecol. Manage. 258, $2009,178-187$.

Liu J.G., Integrating ecology with human demography, behavior, and socioeconomics: needs and approaches, Ecol. Modell. 140, 2001, 1-8.

Lunney D., Pressey B., Archer M., Hand S., Godthelp H. and Curtin A., Integrating ecology and economics: illustrating the need to resolve the conflicts of space and time, Ecol. Econ. 23, 1997 , 135-143.

Mac Nally R. and Brown G.W., Reptiles and habitat fragmentation in the box-ironbark forests of central Victoria, Australia: predictions, compositional change and faunal nestedness, Oecologia 128, $2001,116-125$.

Marcot B.G., Steventon J.D., Sutherland G.D. and McCann R.K., Guidelines for developing and updating Bayesian belief networks applied to ecological modeling and conservation, Can_._. For.est _ _Res.

Forestiere 36, 2006, 3063-3074.

Martin T.G., Kuhnert P.M., Mengersen K. and Possingham H.P., The power of expert opinion in ecological models using Bayesian methods: impact of grazing on birds, Ecol. Appl. 15, 2005, 266280. 


\section{ELSEVIER_BIOC_5770}

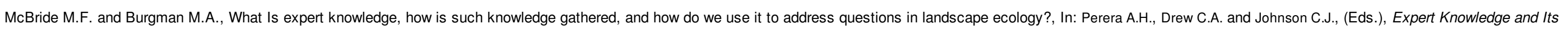

Application in Landscape Ecology, 2012, Springer; New York, 11-38.

McCann R.K., Marcot B.G. and Ellis R., Bayesian belief networks: applications in ecology and natural resource management, Can. J. For. Res. 36, $2006,3053-3062$.

McIntyre S. and Hobbs R., A framework for conceptualizing human effects on landscapes and its relevance to management and research models, Conserv. Biol. 13, 1999, 1282-1292.

Measham T.G., Building capacity for environmental management: Local knowledge and rehabilitation on the Gippsland Red Gum Plains, Aust. Geogr. 38, 2007, 145-159.

Menz M.H., Dixon K.W. and Hobbs R.J., Hurdles and opportunities for landscape-scale restoration, Science 339, 2013, 526-527.

Morcom L.A. and Westbrooke M.E., The pre-settlement vegatation of the Western and Central Wimmera Plains of Victoria, Australia, Aust. Geogr. Stud. 36, 1998, 273-288.

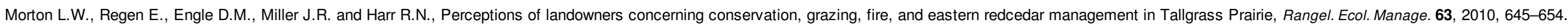
Munro N.T., Fischer J., Wood J. and Lindenmayer D.B., Revegetation in agricultural areas: the development of structural complexity and floristic diversity, Ecol. Appl. 19, $2009,1197-1210$.

Munro N.T., Lindenmayer D.B. and Fischer J., Faunal response to revegetation in agricultural areas of Australia: a review, Ecol. Manage. Restor. 8, 2007, 199-207.

Netica 4.09, 2009. Netica 4.09, Ed. N.S. Corp.

Nichols P.W.B., Morris E.C. and Keith D.A., Testing a facilitation model for ecosystem restoration: does tree planting restore ground layer species in a grassy woodland?, Austral Ecol. 35, $2010,888-897$.

Olsson P., Gunderson L.H., Carpenter S.R., Ryan P., Lebel L., Folke C. and Holling C.S., Shooting the rapids: navigating transitions to adaptive governance of social-ecological systems, Ecol. Soc. 11, $2006,18$.

Pannell D.J., Marshall G.R., Barr N., Curtis A., Vanclay F. and Wilkinson R., Understanding and promoting adoption of conservation practices by rural landholders, Aust. J. Exp. Agric. 46, 2006, 1407-1424.

Pearl J., Probabilistic Reasoning in Intelligent Systems: Networks of Plausible Inference, 1988, Morgan Kaufmann; San Francisco.

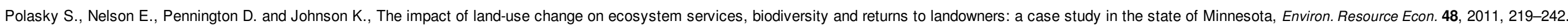
Pollino C.A., Woodberry O., Nicholson A.E., Korb K.B. and Hart B.T., Parameterisation and evaluation of a Bayesian network for use in an ecological risk assessment, Environ Modell Software 22, 2007, 1140-1152.

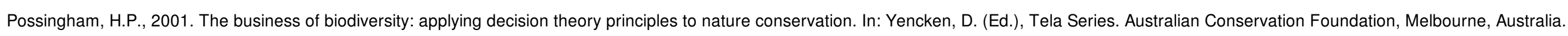
Radford J.Q., Bennett A.F. and Cheers G.J., Landscape-level thresholds of habitat cover for woodland-dependent birds, Biol. Conserv. 124, 2005 , 317-337.

Rumpff L., Duncan D.H., Vesk P.A., Keith D.A. and Wintle B.A., State-and-transition modelling for adaptive management of native woodlands, Biol. Conserv. 144, 2011, 1224-1236.

Rushton, D., 2006. River restoration - how much does it cost?, D.o.E.W. Australia, Department of Environment Western Australia, Perth, p. 18.

Schaffers A.P., Raemakers I.P., Sykora K.V. and Ter Braak C.J.F., Arthropod assemblages are best predicted by plant species composition, Ecology 89, $2008,782-794$.

Schirmer, J., Field, J., 2000. The cost of revegetation. In: Forestry, A (Ed.), ANU Forestry, Canberra, p. 101.

Seale C., Social Research Methods: A Reader, 2004, Routledge; London, New York.

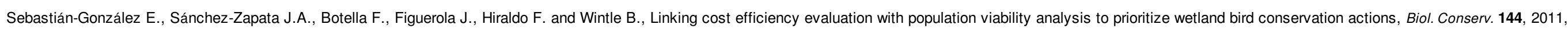
2354-2361.

Segan D.B., Bottrill M.C., Baxter P.W.J. and Possingham H.P., Using conservation evidence to guide management, Conserv. Biol. 25, 2011, $200-202$.

Smith F.P., Who's planting what, where and why - and who's paying? An analysis of farmland revegetation in the central wheatbelt of Western Australia, Landsc. Urban Plan. 86, $2008,66-78$. 


\section{ELSEVIER_BIOC_5770}

Smith T.A., Osmond D.L., Moorman C.E., Stucky J.M. and Gilliam J.W., Effect of vegetation management on bird habitat in riparian buffer zones, Southeast. Nat. 7, 2008, 277-288.

Soulé M.E., Mackey B.G., Recher H.F., Williams J.E., Woinarski J.C.Z., Driscoll D., Dennison W.G. and Jones W.E., The role of connectivity in Australian conservation, Pac. Conserv. Biol. 10, 2004, 266-279.

Soulé M.E. and Sanjayan M.A., Conservation targets: do they help?, Science 279, 1998, 2060-2061.

Speirs-Bridge A., Fidler F., McBride M., Flander L., Cumming G. and Burgman M., Reducing overconfidence in the interval judgments of experts, Risk Anal. 30, 2010, 512-523.

Stern P.C., Dietz T., Kalof L. and Guagnano G.A., Values, beliefs and proenvironmental action - attitude formation toward emergent attitude objects, J. Appl. Soc. Psychol. 25, 1995, 1611-1636.

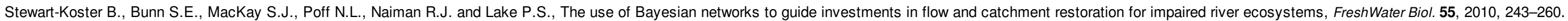
Ticehurst J.L., Curtis A. and Merritt W.S., Using Bayesian Networks to complement conventional analyses to explore landholder management of native vegetation, Environ. Modell. Software 26, 2011, 52-65. Vesk P.A. and Mac Nally R., The clock is ticking - revegetation and habitat for birds and arboreal mammals in rural landscapes of southern Australia, Agric. Ecosyst. Environ. 112, 2006, 356-366.

Walters C., Adaptive Management of Renewable Resources, 1986, Macmillan; New York.

Whittaker D., Vaske J.J. and Manfredo M.J., Specificity and the cognitive hierarchy: value orientations and the acceptability of urban wildlife management actions, Soc. Nat. Resour. 19, 2006, 515-530.

Wyborn C., Jellinek S. and Cooke B., Negotiating multiple motivations in the science and practice of ecological restoration, Ecol. Manage. Restor. 13, 2012, 249-253.

\section{Appendix A. Supplementary material}

\section{Supplementary data 1}

Multimedia Component 1

\section{Highlights}

- Bayesian Networks can predict how social and ecological factors influence biodiversity.

- Faunal gains are greatest when cleared areas are restored compared with remnant and revegetated habitat.

- Cost-effective management actions differ between habitats and faunal groups.

- Landholder demographics can influence habitat variables and faunal species richness.

- Current restoration actions may not increase faunal species in agricultural areas. 